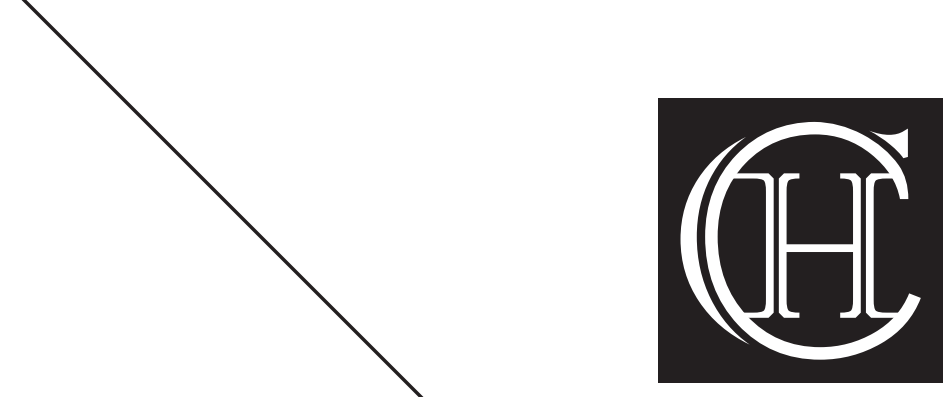

COMUNICACIÓNyHOMBRE

\title{
PRESENTAGIÓN
}

PRESENTATION 

Dra. Daniela Musicco Nombela

Universidad Francisco de Vitoria, Madrid, España

@ daniela.mussico@ufv.es

(iD) $0000-0001-7325-9978$

\section{Data drive / human drive: el reto de la Data Comunicación}

Data drive/ human drive: the challenge of Data Communication

Hoy se generan más de 1700 billones de bytes de datos por minuto. El uso de los Datos en la Comunicación y el posible enriquecimiento que aporta desde el punto de vista del humanismo, es una cuestión que muchos investigadores se han planteado. Serán los Datos quienes nos conduzcan hacia nuevas formas de comunicación o será siempre el hombre quien conducirá la comunicación. ¿Data drive o human drive? Trabajar con grandes cantidades de Datos es lo que ha permitido el desarrollo de la llamada Inteligencia Artificial y su aportación cada vez mayor en el Aprendizaje Profundo, con el uso de redes neuronales. La comunicación llamada Data Comunicación, es aquella basada en el uso de bases de datos: DB y especialmente de BDB, Big Data, relacionada con el Open Data, Company Data, Textual Data... La DATA Comunicación, implica el aprovechamiento de un conjunto de grandes cantidades de datos clasificados y organizados de tal forma que pueden ser encontrados con gran rapidez y facilidad, que pueden llegar a ser datos muy heterogéneos en el caso del Big Data. ¿Cuál es el reto de la Data Comunicación?

PALABRAS CLAVE: data comunicación, data drive / human drive, uso de datos humanidades, datos, datos y comunicación.

Today, more than 1700 billion bytes of data are generated per minute. The use of DATA in the Communication and the possible enrichment that it brings from the point of view of humanism, is an issue that many researchers have raised. Either data will lead us to those new forms of communication or man will always lead the communication. Data drive or human drive? Working with large amounts of data is what has allowed the development of the call based on Artificial Intelligence known as Deep Learning. The communication called DATA Communication, is that based on the use of databases: DB and especially BDB, Big Data, related to Open Data, Company Data, Textual Data ... DATA Communication implies the use of a set of large amounts of classified and organized data in such a way that they can be found very quickly and easily, which can become very heterogeneous data in the case of Big Data. What is the challenge of Data Communication?

KEYWORDS: data communication, data drive / human drive, use of humanities data, data, data and communication. 


\section{Introducción}

A través del uso de Datos en la propia investigación algunos ven la ruptura de la frontera entre estudios cuantitativos y cualitativos, superándose así la fábula de Bertrand Russell sobre el pavo que de forma inductiva llegó a la concusión, tras estar una gran cantidad de días observando el mismo hecho, que dado que todos los días comía a las 9, estaba claro que a las 9 siempre comía, hasta que: «la víspera de Navidad, en vez de darle comida, le cortaron el cuello» (Russell, 1982/2013).

La muestra cuantitativa que puede alcanzarse gracias al big data es tan grande (puede llegar al 100\%), que se hace más fácil aventurar hipótesis en el ámbito social humano relacionándolas con estudios cualitativos. Así que aunque el pavo seguirá sin saber por qué una víspera de Navidad no comió, tal vez podría haber comprendido y relacionado muchas más cosas los días previos a que le cortaran el cuello.

Los Datos son la reproducción simbólica, de un atributo o variable cuantitativa o cualitativa; según la RAE «Información sobre algo concreto que permite su conocimiento exacto o sirve para deducir las consecuencias derivadas de un hecho». Etimológicamente proviene del latín datum: lo que se da. Actualmente los datos se relacionan con bases de datos, bancos de datos, procesamientos de datos...

El uso de Datos en la Comunicación, en un primer enfoque, alejaría la comunicación de vertientes que implican un desarrollo comunicativo basado en las emociones y en las libres y directas percepciones humanas, más ligadas a los sentidos; el almacenamiento de los Datos y el uso de los mismos se asocian a grandes máquinas que sustituyen al cerebro humano y a aspectos más intuitivos, afectivos y espirituales del hombre.

En un segundo acercamiento, sin embargo, el uso de Datos en la Comunicación podría presentar las claves para un nuevo impulso a la razón humana, la emoción, la intuición y la búsqueda de un sentido vital, de la verdad.

Los Datos pretenden aportar fiabilidad, transparencia y la persecución positiva de nuevos puntos de vista, de cambio, no solo de ideas y planteamientos, sino también, a través de esa mayor profundización, de una nueva implicación con la realidad circundante y una novedosa llamada a la acción de búsqueda.

Los Datos son números, pero los números no son solo estadísticas, porcentajes o escalas, corroboran sospechas, intuiciones humanas, dudas, equilibrios y desequilibrios.

Parece factible que a través del big data pudiera ser cierta la posibilidad que planteaba el sociólogo William Bruce Cameron:

Sería bueno si se pudieran enumerar todos los datos que requieren los sociólogos porque entonces podríamos ejecutarlos a través de máquinas IBM y dibujar gráficos como lo hacen los economistas. Sin embargo, no todo lo que se puede contar cuenta, y no todo lo que cuenta se puede contar (Bruce Cameron, 1963).

Aunque tal y como termina la cita volvería a acercarse a la idea de que los Datos nunca podrán dominar la realidad y aún menos la humana.

Basada en la vida real del matemático John Forbes Nash, en la película Una mente 
maravillosa, el investigador dice que «Es solamente en las misteriosas ecuaciones del amor donde se puede encontrar toda razón lógica. Yo estoy aquí gracias a ti» (Beautiful mind, 2001).

Desde Da Divina proportione, el tratado de Luca Pacioli de 1509 sobre las aplicaciones del número áureo, «llamada así por sus propiedades excelsas, supremas, excelentísimas, incomprensibles, inestimables, innumerables, admirables, inefables, singulares ..., que corresponde por semejanza a Dios mismo» (Pacioli, 1509/2017), de la sección aúrea, a los números irracionales como el número $\mathrm{Pi}$, los números, las matemáticas, los algoritmos y los datos, finalmente tienden a rebelarse a la propia lógica y racionalidad. Se alejan de la definición del profesor de informática Ricardo Peña «Conjunto de reglas que, aplicada sistemáticamente a unos datos de entrada apropiados, resuelven un problema en un numero finito de pasos elementales» (Peña Marí, 2006), y están más cerca de Srinivasa Ramanujan el matemático creyente, que decía que sus teoremas eran de inspiración divina «no soy yo quien piensa estas fórmulas» (The man who kew infinity, 2015).

Se habla de periodismo de datos, de data advertising, de data content y de comunication management, data-driven marketing...

El uso de los Datos abre nuevas posibilidades de pasar de lo general a lo particular. Es curioso que tras los algoritmos, los números infinitos, se esconda la oportunidad de llegar al UNO, al único e intransferible. Leon Lederman, el físico premio Nobel, en su libro dedicado a la partícula divina, así lo describió: «esta historia es sobre el universo y, desafortunadamente, no hay Datos sobre el Principio. Ninguno, cero. (...) Cuando lees o escuchas cosas sobre el nacimiento del universo, se trata de invenciones. Estamos en el dominio de la filosofía. Solo el Todopoderoso sabe lo que sucedió al Principio» (Lederman y Teresi, 1993/2012).

La inequívoca inaprensible verdad de lo intangible que encuentra una razón de ser de las cosas, de los números, de los datos, que es independiente y ajena a la propia realidad de los mismos. Así lo expresó el matemático británico Marcus du Sautoy «Soy un platónico de corazón y que creo que encontrarás que la mayoría de los matemáticos lo son. Creo que 17 es un número primo, independientemente de si hay seres humanos cerca que demuestren ese hecho» (Sautoy, 2018).

La Data Comunicacion marca la frontera entre la comunicación de masas, de los mass media y la comunicación personalizada, conectada por la Web y por las redes sociales.

Regresamos a las pequeñas aldeas de la comunicación primitiva del tú a tú. Una especie de vuelta atrás en una máquina del tiempo que ya predijo el visionario científico y espiritual Marshall MacLuhan: la aldea global «La nueva interdependencia con la electrónica reproduce un mundo a imagen de una aldea global» (McLuhan, 1964).

Los datos encuentran nuestros perfiles, nuestros intereses, nos nominalizan, nos vuelven a poner un nombre, un rostro y por qué no: un corazón y un alma.

Nada más lejos de los números tatuados en los brazos de los condenados judíos: «al hombre del lager se le puede quitar todo, excepto una sola cosa: la última libertad humana de enfrentarse espiritualmente, de una forma u otra, a la situación impuesta" (Frankl, 1946/2015). En la misma línea de aquello que se "escapa" a los datos, Javier Prades en La razón ¿enemiga del misterio?, plantea la historia de aquel director general que no pudiendo ir a un concierto de la Sinfonía $n^{\circ} 8$ Inacabada, de Schubert, regala su entrada al jefe de personal y éste, creyendo que debía seguir haciendo su trabajo incluso como espectador, 
después del concierto le entrega un informe, intentando optimizar los números y aportando datos concluyentes,

1. durante considerables períodos de tiempo los cuatro oboes no hicieron nada, debería reducirse su número y distribuir su trabajo entre el resto de la orquesta, eliminando con ellos picos de empleo;

2. Ios doce violines tocan la misma nota, por lo que la plantilla de los violinistas debería reducirse drásticamente;

3. no sirve para nada que los instrumentos de viento repitan sonidos que han sido ya interpretados por las cuerdas;

4. si tales pasos redundantes se eliminasen, el concierto podría reducirse a un cuarto;

5. si Schubert hubiese tenido en cuenta estas indicaciones, habría terminado la sinfonía (Prades, 2007).

Datos y números contrapuestos a la emoción, al pensamiento del hombre, amenazando su libertad, así lo decía Orwell en 1984: «Después de todo, ¿cómo sabemos que dos más dos son cuatro? ¿O qué la fuerza de gravedad realmente existe? ¿O que el pasado es inmutable? ¿Qué pasa si el pasado y el mundo exterior existen solo en tu mente y tu mente está bajo control?» (Orwell, 1948/2016).

Se ha temido siempre el mundo de los datos como un mundo que nos aleja de la humanidad, del humanismo, pero eso no es cierto o no tiene porqué.

El humanismo tiene mucho que hacer en este campo Data, pero debe actuar, entrometerse, comprender, estudiar, analizar y finalmente jugar a favor.

\section{Objetivo y metodología}

En el número 16 de la revista de investigación científica Comunicación y Hombre, el objetivo principal ha sido encontrar los nexos de unión entre los diferentes usos de los Datos en la Comunicación. Se ha mantenido el enfoque interdisciplinar acercando áreas de estudio relacionadas con los Datos.

Para llevar a cabo la elaboración del número 16 se ha utilizado la misma metodología empleada en números anteriores: se ha partido de un primer acercamiento al tema teórico; posteriormente se ha iniciado la búsqueda de autores de habla hispana o que publiquen en español (dado que hasta el momento aún no se trata de una publicación bilingüe al 100\% sino en proceso de serlo) sobre el tema de estudio propuesto de la DATA COMUNICACIÓN; después de una primera lectura de sus trabajos se les ha propuesto participar en el número de la revista y realizar una nueva aportación científica ad hoc, inédita, sobre el tema central, sometiéndose posteriormente a la doble revisión ciega por pares; a la vez tras el call for paper, se han recepcionado artículos que han llegado respondiendo al tema propuesto y que también han sido sometidos a la doble revisión ciega por pares.

Además, como en otros números se han puesto en diálogo artículos sobre comunicación 
y humanidades con un tema en común y buscando nexos con otras disciplinas.

Se han buscado investigadores tanto a nivel nacional como internacional.

En el ámbito nacional colaboran investigadores de estas universidades:

Universidad de Cádiz .2

Universidad Rey Juan Carlos...............................................

Universidad Complutense.................................................6

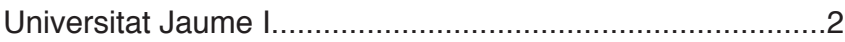

Universidad de Castilla La Mancha.....................................2

Universidad Politécnica de Madrid.......................................2

Hospital Universitario Puerta de Hierro..............................2

Universidad Carlos III de Madrid............................................1

Universidad CES Don Bosco (Complutense).......................1

Universidad Camilo José Cela............................................3

Universidad Autónoma de Madrid........................................1

Universidad de Navarra.................................................

Universidad de Huelva ......................................................1

Universidad Francisco de Vitoria.............................................4

Universidad CEU San Pablo Madrid.....................................

En el ámbito internacional están representados los siguientes países y universidades:

Colombia Universidad del Valle.........................................1

Colombia Universidad Distrital Francisco José de Caldas.....2

Hungría Budapest University of Technology and Economics..1

Méjico Universidad Tecnológico de Monterrey......................2

Méjico Universidad Nacional Autónoma de México.................1

Bolivia Universidad Técnica de Oruro.....................................1

Argentina Universidad Nacional de Córdoba.........................1

Argentina Universidad Nacional de Villa María......................1

Las áreas de estudio y disciplinas abordadas son: la comunicación de empresa, la publicidad, la información periodística, la medicina, el deporte, la empresa, la música, el arte...

\section{El reto de la Data Comunicación}

Los datos pueden aportar rigor, conocimiento y compromiso. Ya no hay quiebros, ni sombras, ni grises. Las cosas son o no son.

A la vez los Datos contemplan infinidad de matices en los que encontrar unidades complejas. No es necesario simplificar y se pueden estudiar infinidad de cosas incluso relacionadas con lo intangible, tan inmateriales como convertir en científico el análisis de las consecuencias del amor sobre el ser humano.

En este número de Comunicación y Hombre se ha hecho un llamamiento para recoger 
artículos que abordan desde diferentes áreas del conocimiento el uso de Datos, y en nosotros queda abierta la oportunidad de activar la relación de la Data Comunicación con la más profunda esencia humana.

Así se expresa en el libro El sentido busca al hombre donde podemos encontrar que de hecho, las realidades que más nos importan no son tanto aquellas cuya fórmula matemática o composición química podemos dar, sino estas que escapan sin cesar a una reducción semejante. No muchos están dispuestos a vivir y a morir por una suma aritmética, por muy verdadera que sea, pero sí por mantener vivo el recuerdo de la persona amada, o por los valores en los que cree, aunque su "definición científica" sea muy defectuosa o no pueda probarlos empíricamente (Antuñano, Sánchez, Huvelle, 2014).

\subsection{Investigadores en diálogo con la Data Comunicación desde DIFERENTES DISCIPLINAS}

Desde diferentes disciplinas tanto en el ámbito nacional como en el internacional, numerosos investigadores están trabajando en el campo del uso de Datos.

Así ocurre en las investigaciones del uso de Datos en la construcción de relatos, dando lugar al Data Storytelling, tal y como analizan los investigadores Lucia Caro-Castaño y David Selva-Ruiz en su artículo Data storytelling: el empleo de datos en la construcción de relatos publicitarios de marca para la revista Comunicación y Hombre. Es interesante la reflexión que los autores hacen sobre como las campañas que usan Datos, deben ponerlos en valor para la emoción humana, convertirlos en una representación emotiva, para poder emocionar y conmover al público así por ejemplo, la campaña Most Dangerous Street, de FCB Chicago para el Illinois Council Against Handgun Violence, intenta concienciar acerca del hecho de que se dispara a 40 personas al día en Chicago. Mientras que este dato frío y disperso a lo largo de la ciudad no resulta significativo para el ciudadano medio, condensar la representación de los 40 disparos en una misma calle resulta muy impactante.

La relación de Datos y música es abordada en la investigación Reconocimiento de emociones musicales a través de datos y tecnologías digitales, en la que los autores Roberto y Juan David Luján Villar escriben para la revista Comunicación y Hombre los resultados de su investigación acerca del uso de Datos para conocer la incidencia de la música en la experiencia emocional de la persona, tal y como se muestra en la figura 4 que representa un experimento realizado con la canción rap Una para Hakeem del rapero R-Two, donde se muestran los tránsitos anímicos de la canción analizada. En el artículo se hacen notar los alcances de la metodología REM (Music Emotion Recognition MER) y sus nuevos puntos de utilidad de desarrollo.

Manteniendo la investigación en el campo de nexos de unión de uso de Datos y de reconocimientos de emociones e intereses culturales, el artículo de los autores Ruth Fernádez-Hernández, Trinidad Vacas-Guerrero y Fernando García-Muiña sobre cómo los gestores de instituciones museológicas pueden hacer uso de la enorme cantidad de Datos que pueden recabar de su público, para conocerlo mejor y para la toma de decisiones. Así el artículo, La llegada del Data a los museos, para Comunicación y Hombre, abre una nueva relación del uso de las tecnologías en los museos y la recopilación de Datos como un campo 
de trabajo diferente: La recolección de datos en los museos y en las redes sociales asociadas y su estudio a través de las nuevas herramientas tecnológicas conlleva un conocimiento nuevo. El ejemplo del Museo Reina Sofía en colaboración con Telefónica y Synergic Partners es uno de los puntos de inicio.

En el campo del periodismo también los Datos pueden ser un instrumento de apoyo notable en el propio trabajo de investigación periodística y en la búsqueda de la verdad. Existe ya un movimiento de estudio y desarrollo del llamado Periodismo de Datos y los datos comienzan a ser un ancla contra las corrientes que arrastran la información mar adentro, hacia el océano abismal de las fake news. Jesús Miguel Flores Vivar, lo muestra en su estudio sobre Datos masivos, algoritmización y nuevos medios frente a desinformación y fake news. Bots para minimizar el impacto en las organizaciones, realizado para el $n^{\circ} 16$ de la Revista Comunicación y Hombre. Plantea de qué forma se está iniciando una autodefensa ante la desinformación. Muchas de estas iniciativas recalan en la inteligencia artificial que con el arte de los algoritmos desarrolla netbots y plataformas con el objetivo de luchar contra la toxicidad de la información. En este sentido una vez más se pone a la luz que no es suficiente el uso de nuevas tecnologías y el recabar Datos, el papel primordial lo tienen las personas capaces de analizar los profesionales que ostentan un perfil multi-interdisciplinar con capacidad para utilizar diversas técnicas de big data y herramientas de filtrado y visualización de datos. Este perfil multi-interdisciplinar no proviene solamente del mundo propiamente de los Datos, de la informática, de la física o las matemáticas, capaces de crear los llamados bots buenos para luchar contra los bots dañinos, sino también personas formadas humanísticamente con capacidad de raciocinio, entendimiento, discernimiento entre fuentes, números, datos y contenidos e interesadas en un valor ético de la búsqueda de la verdad.

En el área de investigación del uso de los Datos en la Comunicación corporativa, se encuentra un campo de estudio en crecimiento; entre las muchas facetas de cambio se encuentra la facilidad de la microsegmentación que aporta el big data, y que favorece el desarrollo de la información relevante, abriendo nuevas posibilidades a la comunicación empresarial. Tras una investigación cualitativa con entrevistas a los responsables de comunicación de empresas nacionales e internacionales con sede en España, las autoras Susana Miquel Segarra y Cristina Aced Toledano, en su artículo Big data: la revolución de los datos y su impacto en la comunicación corporativa, presentan unos resultados concluyentes: muestran una necesidad de compromiso, implicación y formación de los profesionales, aśi como la importancia de que tengan una especial sensibilidad con el uso de los datos.

Alejándonos de la Comunicación y buscando los nexos interdisciplinares de otros campos de estudio del uso de Datos, encontramos que el sector deportivo es otro de los campos de desarrollo en crecimiento; los investigadores Leonor Gallardo y Jorge García-Unanue, presentan su interesante trabajo en su artículo Los datos, las analíticas y la digitalización como claves del futuro en la Gestión Deportiva pública y privada, en el que se aborda el tema de estudio en relación con el desarrollo competencial y la gestión deportiva. Se trata de un artículo que muestra los resultados de dos proyectos de investigación en torno al uso de Datos y deporte; el primero es un proyecto de investigación financiado por la segunda convocatoria de Ayudas José María Cagigal, otorgado por la Federación de Asociaciones de Gestores Deportivos Española (FAGDE); el segundo es un proyecto europeo de innovación y desarrollo en el marco de la convocatoria del Programa Erasmus + KA203 de Asociaciones 
Estratégicas para la Educación superior, realizado por un consorcio de nueve universidades de diferentes países de la Unión Europea. Concluyen los autores en el primero que el uso de big data es la herramienta que requiere un mayor desarrollo para el futuro éxito de las organizaciones deportivas y en el segundo que en la actualidad hay una gestión del datos por debajo de la media, siendo sin embargo las que requieren un mayor desarrollo para el futuro profesional de los gestores deportivos.

El análisis de los Datos de las historias clínicas digitales, va a ser uno de los campos de mayor interés en la investigación, dada su relación directa con la posibilidad de dar un avance no solo al conocimiento y estudio de las enfermedades sino sobre todo por el impulso al descubrimiento de nuevas formas de curación y de prevención de las mismas. Es necesaria la cobertura de todos los datos posibles para desarrollar nuevos servicios con el objetivo de mejorar el seguimiento y la prevención de enfermedades, y generar valor a partir de ellos. Las enfermedades como el cáncer, tal y como explican, Ernestina Mensalvas, Alejandro Rodríguez-Gonzalez, María Torrente, y Mariano Provencio, autores del artículo ¿Puede data science ayudarnos a mejorar el pronóstico y tratamiento del paciente oncológico?, sin duda encuentran un gran beneficio en la investigación a través de las técnicas de big data, integran múltiples fuentes de datos de forma que se puede analizar datos no estructurados, notas clínicas y literatura; extraer patrones para predecir toxicidad, o efectos adversos e interacciones de medicamentos a gran escala.

Volviendo al campo de la Comunicación y el uso de Datos, otro aspecto de gran interés es el presentado en el artículo sobre la Generación de valor a través del uso del Open Data en la comunicación con el ciudadano. En este artículo los investigadores Guadalupe AguadoGuadalupe y Alberto García-García, toman el caso de la Biblioteca Nacional de España y la Junta de Castilla y León. Se ha observado que la riqueza que aporta el uso de datos está en relación con el tipo de filtrado que se realiza, si bien se constata que predominan elementos como la personalización e interacción con los usuarios para la generación de valor. Entre las muchas aportaciones de la investigación destaca la reflexión compartida por una hipótesis de partida de que en el entorno actual de datos masivos se ha ido dejando de ver el mercado como un conjunto de segmentos delimitados por factores de carácter demográfico y geográfico, para verlo como individuos dentro de un entorno donde la interacción con el usuario es un factor determinante. Esta recuperación del individuo hace vislumbrar el posible resurgir de una nueva dimensión de relación humana que enlaza una vez más con el visionario Marshal MacLuhan.

El uso de Datos para evaluar las competencias emprendedoras del alumnado universitario puede ser decisivo para enfrentar las nuevas generaciones a las tendencias de una economía cada vez más necesitada de emprendedores, un activo que debe cultivar, motivar, formar e incentivar cada país. La creación de un instrumento para validar las competencias es de gran utilidad y ha sido el trabajo de investigación del artículo Diseño y validación inicial de un instrumento de medición de la competencia emprendedora sobre su tratamiento y comunicación en las aulas universitarias. Datos preliminares, realizado por los investigadores Guillén Tortajada, Jiménez-Martinez, Szalai, Caballero-García y Alcaraz-Rodríguez y que plantea la importancia de la intervención para el desarrollo de la competencia emprendedora en la Universidad. Los Datos y la medición y análisis de los mismos muestran un complemento positivo, un instrumento de apoyo también en la educación y formación. 
Pero no siempre el uso de Datos o el masivo uso de estos aporta algo positivo; es el caso del ámbito de la política. El Big Data ocupa hoy todos los espacios en que se resuelve la vida en nuestras sociedades. Desde hace algunos años pasó a definir también el plano de la política, modificando profundamente las reglas de juego y poniendo en tela de juicio la legitimidad de las democracias por la injerencia desmesurada del big data en los procesos políticos, en especial los electorales, así se muestra en el artículo El uso del big data en política o la política del big data, de los investigadores Ardini y Nahúm Mirad.

Siempre en el ámbito de la investigación sobre la política es interesante también la contribución del investigador Rafael Martinez Rivas, que logra en su artículo El arte y lo político en Mateo Maté: un estudio de caso, un nexo de unión interdisciplinar entre política y arte en el que se analiza una obra, instalación, en el que un mapa de Europa vallado, mantiene a los individuos separados de sus semejantes, de ahí su nombre Área restringida (Maté, 2007). Concretamente, dado que el espectador se sitúa fuera de un mapa, se puede pensar que la obra habla de la división entre los sujetos que viven dentro del mapa, de la legalidad, de la comunidad, y los extranjeros, los ilegales, los extracomunitarios. El arte, como en el caso de las campañas publicitarias estudiadas en el artículo sobre Data Stoytelling, de Caro Castaño y Selva Ruiz, puede devolver al hombre la experiencia vivida de forma personal y haciendo sensibles los Datos. Tal y como ilustran en su artículo analizando como los números de inmigantes o de asesinatos, pueden ser digeridos de forma mucho más rápida que una vivencia personal a través del arte o de la publicidad, especialmente si estas plantean obras de Street Marketing, Performances o Instalaciones.

Los Datos son el apoyo a la investigación de Orlando Valdez-López, Luis M. Romero-Rodríguez y Ángel Hernando Gómez, autores del artículo La tabloidización y espectacularización mediática: discusión conceptual y aproximaciones empíricas, que se sirvieron de Data Base. Para ello se realizó una criba que partió de criterios, descriptores y palabras clave en las principales bases de datos internacionales (JCR y Scopus) utilizando los algoritmos booleanos AND/OR, de la cual emergieron 52 publicaciones en la colección principal de la Web of Science (Core Collection) y 72 en Scopus. La Investigación muestra un análisis crítico sobre la presencia cada vez mayor del sensacionalismo que doblega la información al utilitarismo de conseguir unos números, unos Datos de audiencia, buscados por medios de comunicación cada vez más enfocados al mercantilismo y que promueven el infoentretenimiento, género que se basa en la información banalizada y el sensacionalismo como anzuelo para aumentar los rating de audiencias.

La desvirtualización de la capacidad humana de comunicar, de informar por la pura persecución de un bien común, de una búsqueda de la verdad, muestra en algunos casos el deterioro moral y ético de las relaciones interpersonales, lugar primordial y apremiante de investigación para futuros estudios.

El artículo La Comunicación Interpersonal y la Comunicación Interna en las empresas: un análisis desde la profesión y la Universidad, de los investigadores Mónica Viñaras Abad, Juan Enrique González Vallés y Silvia Rincón, da una muestra de la acuciante responsabilidad de estudio en el campo de la comunicación interpersonal también a nivel de empresa. El uso de los Datos en la comunicación corporativa ha presentado unos avances que aún están en crecimiento y perfeccionamiento como por ejemplo en el caso de las herramientas de escucha y los datos que estas pueden aportar en el ámbito de la Comunicación Interna de 
empresa.

Regresando a los nexos de unión interdisciplinares del uso de Datos en diferentes áreas de investigación y ámbitos laborales de estudio, es de vital interés de qué forma se están empleando en los avances en el ámbito de la ciencia en general y de la medicina en particular, tal y como se ve en el artículo del investigador Roger Ruiz Moral; si por un lado es notable el avance y la ayuda que aporta a la investigación en medicina el Data Science, no hay que olvidar, al igual que ocurre en otros ámbitos de la Comunicación como la Interna de empresa, la extrema importancia de la relación interpersonal con el paciente; en este sentido la Investigación Consideraciones epistemológicas, antropológicas, éticas y educativas para una Comunicación Clínica Centrada en la persona, presenta un trabajo sobre la CCCP y la práctica de la medicina; si bien suelen ser consideradas desde aproximaciones eminentemente pragmáticas, conllevan presupuestos que trascienden las verdades de tipo científico y considera al paciente como una realidad compleja, única, multidimensional y con potencialidades diversas; en un encuentro clínico que señala el imperativo moral de la medicina (y de la CCCP) de actuar en beneficio del paciente, tal y como explica el autor Roger Ruiz Moral.

Sin duda los Datos representan el deseo humano de búsqueda de respuestas, de verdades, de resolución absoluta de problemas, de control sobre el infinito. Sin embargo parecen encontrar un límite, un límite en la comprensión definitiva de la existencia del hombre, de su corporeidad, de su sentido, de sus emociones y sobre todo de su sufrimiento; en este sentido la investigación La incomunicabilidad del misterio del sufrimiento, de los investigadores Carmen de la Calle Maldonado, Cecilia Castañera Ribé y Pilar Giménez Armentia, atiende a esas preguntas sin respuesta, a esas verdades difíciles o imposibles de comunicar, es posible acompañar y aliviar el sufrimiento del otro pero, al tratarse de un misterio intransferible, nunca una experiencia de dolor puede asimilarse a otra. Estamos ante una realidad en la que cada ser humano se descubre en su verdad y se encuentra consigo mismo de un modo particularmente único. Esta experiencia de incomunicabilidad tan radical exige a la persona la tarea de asimilar, aceptar, vivir y aprender en el dolor, para transformarlo en camino único de sentido y verdadera humanización. Así tenemos que frente al progreso y la gran ayuda que significa el uso de los Datos no hay que olvidar los límites que estos implican. Frente a la idea de que el sufrimiento es un misterio que hay que sobrellevar, la cultura actual (tan condicionada por la mentalidad cientificista dominante y por las promesas trashumanistas de mejora de la condición humana) nos hace creer que el progreso científico y tecnológico, nos permitirá en el futuro evitar la enfermedad y el dolor, alargar la esperanza de vida hasta lo inimaginable e incluso controlar la muerte, tal y como indican los investigadores de la Calle Maldonado, Castañera Ribé y Giménez Armentia.

Si el hombre encuentra en sí mismo el propio límite y en los demás puede hallar una parte de su infinitud, volviendo a los artículos sobre el arte y lo político o sobre la representación emotiva de los datos en el Data Storytelling, capaz de ser compartidos y de con-mover, constituyendo ese todo único que trasciende el individuo limitado en comunidad infinita, la Investigación de los autores Daniel de la Rosa y Silvia Carrascal, Educación para el Desarrollo Sostenible: un estudio sobre la difusión y contextualización de la educación en los medios de prensa escrita en España: El Mundo, $A B C$ y La Vanguardia, cierra las investigaciones con un objetivo final: el estudio del papel de los medios de comunicación a la hora de transmitir los 
Objetivos de Desarrollo Sostenible (ONU 2015-2030) y de humanizar los datos y empatizar con los lectores a favor de la consecución de las metas de una agenda que se posiciona en la actualidad como la materialización moderna de la Declaración Universal de los Derechos Humanos; esta investigación es el colofón final que atañe a Datos y a humanización de los mismos para poder de nuevo conmover, mover a la acción a través de la Educación para el Desarrollo Sostenible.

Antes de cerrar este epígrafe dedicado al cuerpo de contenidos en diálogo sobre el tema propuesto Data Comunicación del n 16 de Comunicación y Hombre, repasaremos brevemente algunos de los textos reseñados por investigadores del área de la comunicación y de las humanidades. El texto del Dr. José Ángel Agejas sobre el Ibro Hablemos del suicidio de Gabriel Gónzalez Ortiz, abre un punto de reflexión sobre el tema abordado en el artículo sobre la Incomunicabilidad del sufrimiento recogido en este mismo número de la revista; probablemente uno de los temas tabú en la comunicación de finales del siglo XX y principios del XXI sea precisamente, con la enfermedad, la muerte y el suicidio como uno de los más censurados. El autor del libro también hace uso de los Datos para poner al lector ante una realidad ocultada. Tal como dice el autor de la reseña, en un mundo hiperconectado por las redes sociales, en el que Google arroja millones de resultados de sitios web con contenidos peligrosos para personas en riesgo de suicidio, el silencio no parece el comportamiento más responsable.

En relación con el área de estudio de la publicidad el Dr. Raúl Anthony Olmedo Neri realiza una reseña sobre un texto que tiene el interés de rescatar la investigación sobre la publicidad de un carácter puramente técnico y pragmático, el investigador alude a cómo el libro El poder de la industria publicitaria en México, de la Dra. Carola García Calderón, adquiere relevancia en el plano de la investigación ya que se constituye como un referente teórico desde el campo disciplinar de la Sociología.

También dedicada a la publicidad es la reseña del Dr. Francisco Cabezuelo Lorenzo sobre el libro El negocio publicitario en la sociedad digital de la Comunidad Valenciana, de la Dra. Cristina González Oñate; en la reseña se hace referencia al gran cambio de la publicidad en la era digital. El libro analiza los servicios que ofertan las agencias de publicidad haciendo especial hincapié en los cambios producidos por la digitalización.

El Dr. Cruz Javier Rodríguez Acevedo propone una reseña sobre el libro La guerra de Don Manuel, de Manuel Guerra Gómez donde se muestra su experiencia vital en un mundo globalizado y donde se tocan algunos de los temas de estudio que han interesado al autor: las sectas, la masonería, la secularización y el laicismo, han creado una serie de discrepancias desde la Revolución francesa hasta nuestros días, que comienzan a fraguarse a partir de la Reforma protestante.

Volviendo a temas relacionados con la información y la desinformación, la reseña Infoética. El periodismo liberado de lo políticamente correcto, del Dr. Álvaro Abellán-García Barrio sobre el libro del profesor Gabriel Galdón López, que actualiza su anterior texto, Desinformación. Métodos, aspectos y soluciones, ilustra una realidad y muestra a su vez soluciones. Según aparece reseñado, el libro es valiente en el posicionamiento y como dice el autor de la reseña Galdón habla fuerte y claro, abordando temas raramente relacionados con la información como los que aborda. En la tercera parte, Galdón nos habla de «verdad y amor en la mirada» (p. 221) como lo característico de la mirada sabia. Esta mirada proporciona sabiduría sobre 
la realidad, «según su jerarquía humana».

Finalmente el libro Los asquerosos de Santiago Lorenzo, es la reseña que propone la Dra. Carmen Fragero. Señala el tema principal del libro, la descripción de un hombre deshumanizado, aislado y autosuficiente. En Los asquerosos, el autor critica a cierta parte de sla ociedad aferrada al consumo, olvidada del valor de su tiempo, esclavizada por su trabajo, vulgar y dispuesta a aparentar; aquella que ama la naturaleza, pero consiente que los suyos la destruyan; aparenta cultura, pero solo es palabrería ostentosa.

Con esta última reseña y esta visión de un hombre limitado en sí mismo, se puede hacer el cierre del $n^{\circ} 16$ de la revista Comunicación y Hombre; con esta idea de hombre egoísta, individualista, no emotivo, proclive a reproducir un temido fin de la humanidad al más puro estilo de la distopía de Aldous Huxley, donde de forma triunfal se afirmaba que no se había ahorrado ningún esfuerzo en conseguir preservar la sociedad que habían creado del sentir algún tipo de emoción (Huxley, 1932/2014). No son los Datos, ni la búsqueda de la mirada amorosa más allá de los mismos, ni los hombres inquietos en tratar de contar lo incontable, de atrapar el infinito a través de un big data, ni los rebeldes que piden el contacto humano por encima de cualquier número para resolver cualquier problema... tal vez ese hombre autosuficiente, que no se pregunta, que no mira, que no cuenta, que no ve al otro, pudiera ser él, uno, de los principios de un fin.

\subsection{El análisis de Datos}

Si el uso de Datos está cada vez más difundido, el aprendizaje del análisis de los mismos, es una necesidad imperante.

El análisis de datos ha entrado a formar parte de los programas de estudio en la mayor parte de las universidades del mundo y ha irrumpido con fuerza en la universidades españolas. El Learning Analytics es una técnica que se fundamenta en el análisis de datos, Big Data, Business Intelligence, Data Storytelling, Data Science, Business Analytics...y muchas de las técnicas que se incluyen en algunos de los textos de los artículos presentes en el $n^{\circ} 16$ de la Revista Comunicación y Hombre para obtener datos en tiempo real sobre una determinada formación.

La sociedad está demandando personas capacitadas para dar sentido a todos los datos.

En España ya están incluidas en los planes de estudios de diferentes grados o incluso constituyen nuevos grados, posgrados, masters en universidades como Comillas, Navarra, Universidad Francisco de Vitoria, Complutense, Autónoma de Madrid, Autónoma de Barcelona, Carlos III, Universidad de la Rioja, entre otras.

A pesar de la formación eminentemente técnica, las preguntas sobre el uso de Datos trascienden la informática, la matemática... necesitan de una educación humanista que ayude a discernir, comprender esos datos, ponerlos en valor y darles un sentido vital humano.

El flujo de datos en internet y en redes es interminable y el peligro del exceso de información es la falta de formación para discernir o dicho como ya anticipó Umberto Eco, internet es peligroso para los ignorantes y útil para los sabios (Eco, 1994/2013). Tal como el pensador vislumbraba, la verdadera educación quizás ya no sea dar más información, sino enseñar a elegir. 


\subsection{LA HUIDA DE LA INFOXICACIÓN}

Toda la gran cantidad de información, de datos que se genera y que se puede almacenar, no sirve de nada si no puede ser analizada y aún menos si no se logra un sentido para su uso. Toda esta disposición de estudio, de comparación, de comprensión y sobre todo de sentido, si no existiera, la humanidad estaría abocada a la infoxicación, un exceso de información desordenada que hace palidecer el Síndrome de Stehendal, que describía él mismo diciendo que «Había llegado a ese punto de emoción en el que se encuentran las sensaciones celestes dadas por las Bellas Artes y los sentimientos apasionados. Saliendo de Santa Croce, me latía el corazón, la vida estaba agotada en mí, andaba con miedo a caerme» (Stendhal, 1817/1990). La infoxicación podría acabar presentando síntomas similares aunque no producidos ante la acumulación de belleza, pero sí similar a una borrachera tan aguda de información desordenada e inconexa, que conllevaría una desinformación, en la que las fake news representarían solo un pequeño accidente de efectos secundarios.

Ante esta visión de un mundo abigarrado de datos e información hasta el exceso, la única huida posible sería aprender a filtrar y decantar, decantar y tal vez quedarse con poco o con nada; el silencio y el vacío como un lugar antitético de necesidad. En este punto se prepara el $\mathrm{n}^{\circ} 17$ de la Revista Comunicación y Hombre: la infoxicación y el vacío, una nueva búsqueda hacia las dos caras de la moneda de la comunicación del siglo XXI, el veneno y tal vez el antídoto.

\section{Conclusiones}

Sin duda la Data Comunicación y el uso de Datos es un nuevo reto para el hombre del siglo XXI.

Su responsabilidad es no rebajar la existencia humana a los números, sino rescatarla de éstos, como en la película Ben-Hur cuando el cónsul romano se dirige al galeote que acaba de salvarle la vida y le dice ¿Cuál es tu nombre, 41?: En ese momento el cónsul ha visto que ese hombre vejado no actuó con despecho, antes le devolvió bien por mal (López Quintas, 2009).

A la vez cultivar el aprendizaje de rescatar los Datos, los números, las fórmulas para su función decisiva de la categoría de relación en la ciencia, tal y como expresa el investigador, doctor en filosofía y académico español Alfonso López Quintás, que también nos sugiere diciendo que un profesor de matemáticas no debe contentarse con enseñar a los alumnos a operar con las estructuras matemáticas: resolver una ecuación, interpretar una fórmula... Al mismo tiempo debe hacerles ver la fecundidad y belleza que tienen tales estructuras, que son tramas de interrelaciones (Quintás, 2009). Tal y como escribe Max Planck, el fundador de la Mecánica Cuántica, que afirma que Kepler se mantuvo fiel a su investigación científica gracias a su fe profunda en la existencia de un plan definido detrás de la creación entera (Wilber, 1987). 


\section{Bibliografía}

ANTUÑANO, Salvador, SÁNCHEZ, Florencio y HUVELLE, Santiago (2014). El sentido busca al hombre. Editorial UFV.

BRUCE CAMERON, William (1963). Informal sociology, a casual introduction to sociological thinking. Random House.

DU SAUTOY, Marcus (2018). Hasta el infinito y más allá. Conferencia, Querétaro, 7 de Septiembre. Universidad Anáhuac, Aula Magna Multiva. Entrevista interactiva con BBC Mundo. Recuperado en https://www.bbc.com/mundo/noticias-45402864.

ECO, Umberto (1994/2013). El exceso de información es malo. Recuperado de http://ssociologos. com/2013/11/03/umberto-eco-el-exceso-de-informacion-es-malo/.

FRANKL, Viktor E. (1946/2015). El hombre en busca de sentido. Herder.

HUXLEY, Aldous (1932/2014). Un mundo feliz. Debolsillo.

LEDERMAN, Leon y TERESI, Dick (1993/2012). La particella di Dio. Se l' universo è la domanda, qual è la risposta?. Focus.

LÓPEZ QUINTAS, Alfonso (2009). Descubrir la grandeza de la vida. Desclée De Brouwer.

McLUHAN, Marshall (1964). Capire i media. Gli strumenti del comunicare. Net.

ORWEL, George, (1948/2016). 1984, Mondadori.

PACIOLI, Luca (1509/2017). Antologia della Divina Proporzione. Aboca.

PEÑA MARÍ, Ricardo, (2006). De Euclides a Java, la historia de los algoritmos y del lenguaje de programación. Nívola.

PRADES, Javier (2007). La razón ¿enemiga del misterio?. Encuentro.

RUSSEL, Bertrand (1912/2013) I problema della filosofía. Feltrinelli

STENDHAL (1817/1990). Roma, Napoli e Firenze. Viaggio in Italia da Milano a Reggio Calabria. Laterza.

WILBER, Kenneth Earl (2017). Cuestiones cuánticas. Kairós. 